\title{
Enhancing the Oil-Fouling Resistance of Polymeric Membrane Ion- Selective Electrodes by Surface Modification of a Zwitterionic Polymer-Based Oleophobic Self-Cleaning Coating
}

\author{
Longbin Qi, " Tianjia Jiang, ${ }^{\#}$ Rongning Liang, and Wei Qin*
}

Cite This: Anal. Chem. 2021, 93, 6932-6937

Read Online

ACCESS | Lلll Metrics \& More | 国 Article Recommendations ｜ sl Supporting Information

ABSTRACT: Due to the frequent oil spill accidents and pollution of industrial oily wastewater, oil fouling has become a great challenge to polymeric membrane ionselective electrodes (ISEs) for applications in oil-contaminated areas. Herein, a simple approach is proposed to enhance the oil-fouling resistance of polymeric membrane ISEs by surface modification of a zwitterionic polymer-based underwater oleophobic coating. As a proof-of-concept, a classical poly(vinyl chloride) membrane-based calcium ion-selective electrode $\left(\mathrm{Ca}^{2+}\right.$-ISE) is chosen as a model sensor. The zwitterionic polymer-based coating can be readily modified on the sensor's surface by immersion of the electrode into a mixture solution of dopamine and a zwitterionic acrylate monomer (i.e., sulfobetaine methacrylate, SBMA). The formed poly(SBMA) (PSBMA) coating alters the oleophilic membrane surface to an oleophobic one, which endows the surface with excellent self-cleaning properties without loss of the sensor's analytical performance. Compared to the pristine $\mathrm{Ca}^{2+}$ -

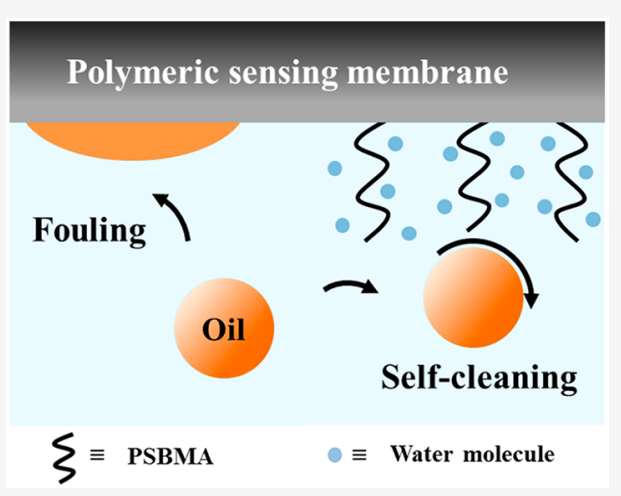
ISE, the PSBMA-modified $\mathrm{Ca}^{2+}$-ISE exhibits an improved analytical stability when exposed to oil-containing wastewater. The proposed approach can be explored to enhance the oil-fouling resistance of other polymeric membrane-based electrochemical sensors for use in the oil-polluted environment.

\section{INTRODUCTION}

As a well-established analytical tool, potentiometric polymeric membrane ion-selective electrodes (ISEs) play an important role in environmental monitoring. ${ }^{1-5}$ Due to the frequent oil spill accidents and increasing discharges of industrial oily wastewater, especially in coastal areas and oilfields, oil foulants are becoming commonplace, ${ }^{6-12}$ which would induce a great challenge for plasticized polymeric membrane sensors to analyze environmental samples with high accuracy and stability. Indeed, oil foulants are found to be much more intractable compared to other foulants (e.g., proteins and microorganisms) owing to their easy deposition and deformation on polymer membranes via strong hydrophobic interactions. $^{13-16}$ When the oil droplets adhere onto a polymeric membrane, they can spread all over the membrane surface in a short time. Moreover, the lipophilic components of the oil droplets could be extracted into the organic membrane phase resulting in a drift in the potential response and loss of the sensor's selectivity. ${ }^{17}$ To date, considerable efforts have been devoted to improve the sensors' biocompatibilities. ${ }^{18-23}$ However, research on the sensors' environmental compatibilities are still rather rare. ${ }^{24-26}$ Considering the negative effects of oil fouling, it is crucial to develop a polymeric membrane ISE configuration with oil-fouling resistance for reliable detection in environmental samples.
Many approaches, such as sample pretreatments (e.g., solvent extraction) and advanced sampling techniques, have been developed to mitigate the interferences of lipophilic compounds. ${ }^{17,27-29}$ However, these methods are usually timeconsuming and unsuitable for the real-time analysis. Surface modification has been proven as an effective method to prevent fouling. ${ }^{30}$ Hydrophilic materials, such as poly(ethylene glycol) (PEG)-based polymers, ${ }^{31,32}$ hydrogels, ${ }^{33}$ nanomateri$\mathrm{als}^{24}$ and zwitterionic materials, ${ }^{34}$ have been employed to protect sensors from direct contact with foulants. Among these, zwitterionic materials are recognized as an excellent candidate for construction of fouling-resistance surfaces due to their electric neutrality, excellent hydrophilicity, underwater oleophobicity, and good stability. ${ }^{13,35-37}$ The unique structures of zwitterionic materials containing equivalent positively and negatively charged groups allow binding with water molecules via strong electrostatic interactions. ${ }^{38}$ Moreover, compared with the PEG-based materials, the zwitterionic units (e.g., sulfobetaine methacrylate, SBMA) could bind to more

Received: March 14, 2021

Accepted: April 20, 2021

Published: April 29, 2021 
Scheme 1. Schematic Illustrations of the Fabrication of the Zwitterionic Polymer Modified Membrane and of the Anti-OilFouling Principle of the Modified $\mathrm{Ca}^{2+}-\mathrm{ISE}$

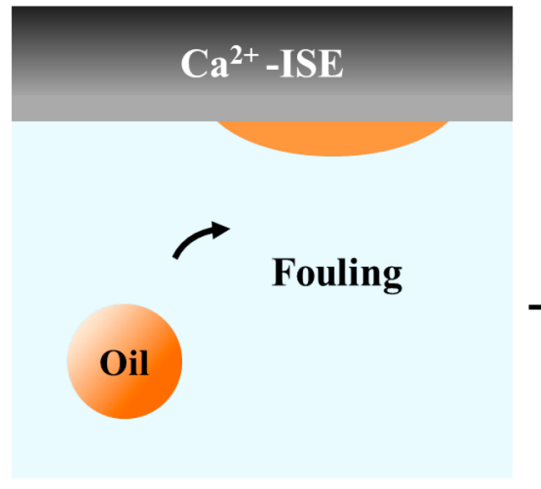

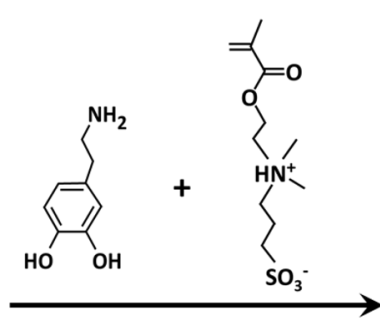

One-step modification

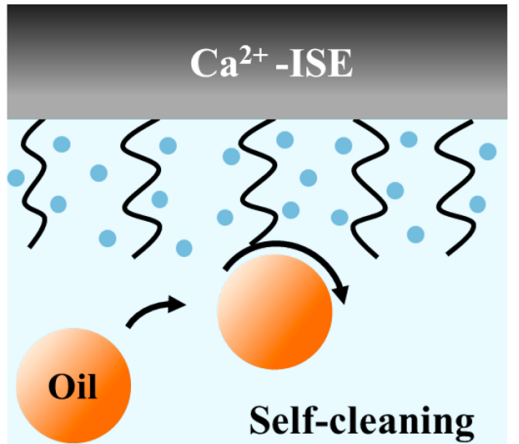

$\zeta \equiv$ PSBMA $\equiv$ Water molecule water molecules (i.e., 7-8 water molecules per unit) and maintain more mobility of water molecules in the hydration layer. ${ }^{39}$ Very recently, the zwitterionic SBMA-based coatings have been modified on voltammetric/amperometric carbonbased electrodes to improve their biocompatibilities. ${ }^{34,40}$ However, it should be noted that these works aimed to develop biocompatible electrochemical sensors which could resist nonspecific protein binding in complex biological fluids. To the best of our knowledge, no research has been done to enhance the oil-fouling resistance of polymeric membrane ISEs for potentiometric detection in the oil-polluted environment.

In this work, for the first time, the influences of oil fouling on the potentiometric polymeric membrane ISEs have been extensively investigated and a promising method is proposed to prevent the ISEs from oil fouling. As a proof-of-concept, a classical poly(vinyl chloride) membrane-based calcium ionselective electrode $\left(\mathrm{Ca}^{2+}\right.$-ISE) is chosen as a model sensor. The surface of the $\mathrm{Ca}^{2+}$-ISE is modified with a zwitterionic poly(SBMA) (PSBMA) coating via a mild dopamine-assisted codeposition method. It will be shown that the PSBMA coating endows the modified $\mathrm{Ca}^{2+}$-ISE with an excellent selfcleaning ability against oil foulants but does not influence the sensor's analytical performance.

\section{EXPERIMENTAL SECTION}

2.1. Membranes and Electrodes. The calcium ionselective membranes ( $\mathrm{Ca}^{2+}$-ISMs) contained $2.3 \mathrm{wt} \%$ $N, N, N^{\prime}, N^{\prime}$-tetracyclohexyl-3-oxapentanediamide (ETH 129), $2.2 \mathrm{wt} \%$ sodium tetrakis [3,5-bis(trifluoromethyl)phenyl] borate (NaTFPB), $31.8 \mathrm{wt} \%$ poly(vinyl chloride) (PVC), and 63.7 wt $\% 2$-nitrophenyl octyl ether (o-NPOE). The membrane cocktail solution was prepared by dissolving 360 $\mathrm{mg}$ of the membrane components into $3.6 \mathrm{~mL}$ of tetrahydrofuran (THF). After being stirred for $2 \mathrm{~h}$, the membrane cocktail solution was poured into a glass ring (i.d. $36 \mathrm{~mm}$ ) fixed on a glass plate to obtain the $\mathrm{Ca}^{2+}$-ISM.

The solid-contact $\mathrm{Ca}^{2+}$-ISEs were prepared as described before. $^{20,24}$ A poly(3,4-ethylenedioxythiophene) (PEDOT):poly(sodium 4-styrenesulfonate) (PSS) layer was used as the ion-to-electron transducer of the solid-contact $\mathrm{Ca}^{2+}$-ISEs. This layer was electrodeposited onto the surface of a glassy carbon electrode (GCE, i.d. $3.0 \mathrm{~mm}$ ) using a mixture solution with $10^{-2} \mathrm{M}$ 3,4-ethylenedioxythiophene and $10^{-1} \mathrm{M}$ PSS. A constant current of $0.014 \mathrm{~mA}$ was applied for the polymerization of $714 \mathrm{~s}^{41,42}$ The obtained GCEs were rinsed with deionized water and dried at room temperature. Finally, $80 \mu \mathrm{L}$ of the membrane cocktail solution was drop-cast onto the GCE to generate a sensing membrane with a thickness of approximately $100 \mu \mathrm{m}$.

2.2. Surface Modification of the $\mathrm{Ca}^{2+}$-ISEs. A one-step deposition method was used to modify the zwitterionic polymer coating onto the surface of the $\mathrm{Ca}^{2+}$-ISE. ${ }^{43}$ Briefly, $2 \mathrm{mg} \mathrm{mL}{ }^{-1}$ of dopamine and $30 \mathrm{mg} \mathrm{mL}^{-1}$ of zwitterionic acrylate monomer SBMA were dissolved into a Tris- $\mathrm{HCl}$ buffer solution $(\mathrm{pH}=8.5,50 \mathrm{mM})$. Subsequently, the membrane electrodes were immersed into the obtained solution and incubated for $12 \mathrm{~h}$ at room temperature. During this process, the self-polymerization of dopamine can trigger the polymerization of the acrylate monomer SBMA to form PSBMA. Simultaneously, the generated polydopamine assists PSBMA to deposit onto the electrode surface. ${ }^{43-45}$ After modification, the obtained electrodes were thoroughly washed with deionized water.

2.3. Electrochemical Measurements. The electromotive force (EMF) values were measured by using an electrochemical workstation (CHI 760D, Shanghai Chenhua Apparatus Corporation, China) at room temperature in the galvanic cell: $\mathrm{Ag} / \mathrm{AgCl} / 3 \mathrm{M} \mathrm{KCl} /$ sample solution/ISM/ PEDOT-PSS/GCE. For $\mathrm{Ca}^{2+}$ detection, the electrodes were conditioned in $10^{-3} \mathrm{M} \mathrm{CaCl}_{2}$ solution overnight before use. Selectivity measurements were carried out by using the separate solution method, ${ }^{46}$ and the electrodes were conditioned in $10^{-3} \mathrm{M} \mathrm{NaCl}$ solution overnight before measurements. The measured EMF values were corrected for the liquid junction potentials, and the ion activities were calculated according to the Debye-Hückel equation.

2.4. Evaluation of the Anti-Oil-Fouling Properties of the PSBMA-Modified $\mathrm{Ca}^{2+}$-ISEs. To evaluate the oil-fouling resistance of the PSBMA-modified $\mathrm{Ca}^{2+}$-ISEs, the potentiometric responses of the electrodes after exposure to oil foulants were tested. The artificial wastewater samples containing 5\% $(\mathrm{v} / \mathrm{v})$ of the oils were prepared. The diesel oil and crude oil samples (collected from the Bohai Sea, China) were selected as the model foulants, since they are typical oil foulants in the environment. The conditioned membrane electrodes were immersed in the oil-containing wastewater samples for $1 \mathrm{~h}$ under continuous stirring. Then, the fouled electrodes were rinsed with deionized water and transferred to the cell for potentiometric measurements. To observe the oil fouling of 
the membrane electrodes, the surfaces of the $\mathrm{Ca}^{2+}$-ISMs were photographed. For easy visualization, the diesel oil sample was stained with Sudan III.

\section{RESULTS AND DISCUSSION}

Due to oil spill accidents and industrial oil pollution, oil fouling is becoming a great challenge for polymeric membrane ISEs to be applied in the environmental fields. Since the plasticized polymeric membrane matrixes are hydrophobic, oil foulants can easily deposit on the sensing membrane via strong hydrophobic interactions, which may cause measurement errors and shorten the sensors' lifetimes. In this study, an underwater oleophobic self-cleaning coating based on a zwitterionic polymer was developed to enhance the oil-fouling resistance of the $\mathrm{Ca}^{2+}$-ISE. As shown in Scheme 1, the proposed surface coating can be formed simply by immersion of the electrode into a solution containing dopamine $(2 \mathrm{mg}$ $\mathrm{mL}^{-1}$ ) and zwitterionic acrylate monomer SBMA (30 mg $\mathrm{mL}^{-1}$ ) at $\mathrm{pH}$ 8.5. After the one-step modification, the oleophilic membrane surface can be changed to an oleophobic one. In this case, the oil droplets adjacent to the sensor surface can be cleaned by the PSBMA coating, thus effectively eliminating the oil-fouling problems (Scheme 1, right). In contrast, for the pristine sensor, the oil droplets could rapidly be adsorbed on the sensor surface and cause sensor failures (Scheme 1, left).

The images of scanning electron microscopy (SEM) show that numerous nanoparticles can be found on the surface of the PSBMA-modified $\mathrm{Ca}^{2+}$-selective membrane (Figure $\mathrm{S} 1$ ). The results of the water contact angle (WCA) measurements illustrate that the WCA of the $\mathrm{Ca}^{2+}$-selective membrane decreases from $86.3 \pm 1.7$ to $38.1 \pm 4.2^{\circ}$ after PSBMA modification, which indicates that the hydrophilicity of membrane surface is significantly improved by the PSBMA coating (Figure S2). The spectra of attenuated total reflectance-Fourier transform infrared spectroscopy (ATRFTIR) show the new peaks at 1726 and $1033 \mathrm{~cm}^{-1}$ for the product from polymerization of dopamine and SBMA (Figure $\mathrm{S} 3$ ), which are attributed to the $\mathrm{O}-\mathrm{C}=\mathrm{O}$ and $\mathrm{S}=\mathrm{O}$ symmetric stretching vibrations, respectively. ${ }^{43}$ These results indicate that the SBMA units have been incorporated into the polymer. Under alkaline conditions, dopamine can be oxidized into dopamine quinone and then form 5,6-dihydroxyindole and 5,6-indolequinone. ${ }^{47}$ These intermediates can be transferred into the semiquinone radical species via the singleelectron exchange reaction and electron resonance, and then further integrated into polydopamine via the radical coupling reactions. ${ }^{47,48}$ Simultaneously, these radicals can also act as reactive species to trigger the free-radical polymerization of SBMA to form PSBMA. ${ }^{43}$ Due to the intrinsic adhesive nature of polydopamine, the PSBMA can be codeposited onto the surface of the substrate via noncovalent interactions, such as hydrogen bonding and $\pi-\pi$ interactions. ${ }^{44,45,49}$

Since the deposition time plays a vital role in the oleophobicity of the prepared PSBMA coating, the influence of deposition time was investigated. As shown in Figure S4, the pristine $\mathrm{Ca}^{2+}$-ISM has a small underwater contact angle (UWOCA) of $25.4 \pm 2.1^{\circ}$ for dichloromethane, indicating its high lipophilicity. As the deposition time increases from 4 to $12 \mathrm{~h}$, the UWOCA of the pristine $\mathrm{Ca}^{2+}$-ISM increases rapidly from $42.0 \pm 1.3$ to $141.5 \pm 3.4^{\circ}$, which is mainly due to the increase in the thickness of the oleophobic PSBMA coating. A further increase in deposition time would not obviously improve the UWOCA. Thus, $12 \mathrm{~h}$ was selected as the optimal modification time. Moreover, several common oils including diesel oil, petroleum ether, and peanut oil were also used to measure the UWOCAs of the PSBMA modified $\mathrm{Ca}^{2+}{ }_{-}$-ISM. As shown in Figure 1, the oleophilic membrane surface becomes

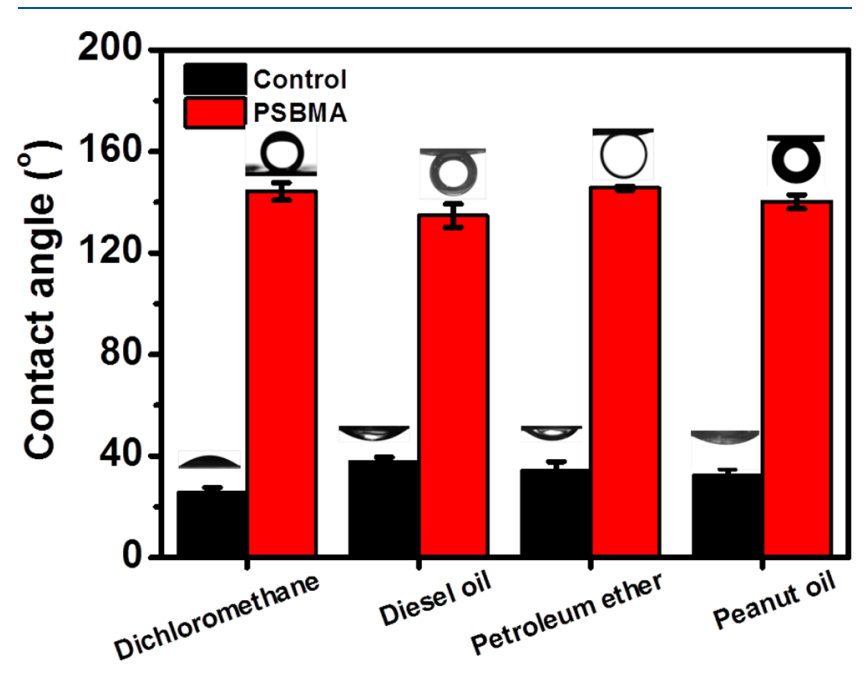

Figure 1. Underwater-oil contact angles (UWOCAs) for the pristine and PSBMA-modified $\mathrm{Ca}^{2+}$-selective membranes. Dichloromethane, diesel oil, petroleum ether, and peanut oil were used to measure the UWOCAs. Experimental conditions: dopamine, $2 \mathrm{mg} \mathrm{mL}^{-1}$; SBMA, $30 \mathrm{mg} \mathrm{mL}^{-1}$; $\mathrm{pH}, 8.5$; immersion time, $12 \mathrm{~h}$.

oleophobic with significantly increased UWOCA values for all of these oils. As a comparison, the UWOCAs for the polydopamine-modified $\mathrm{Ca}^{2+}$-ISM were also investigated (see Figure S5), which are much smaller than those of the PSBMAmodified $\mathrm{Ca}^{2+}$-ISM. These results indicate that the oleophobicity of PVC-based $\mathrm{Ca}^{2+}$-ISE can be significantly enhanced by the proposed PSBMA coating. In addition, the PSBMA coating has an excellent long-term stability. Experiments show that the PSBMA-modified $\mathrm{Ca}^{2+}{ }_{-}$ISM can still maintain a UWOCA value of $142^{\circ}$ for diesel oil after storage at $4{ }^{\circ} \mathrm{C}$ for one month.

The zwitterionic polymers have been recognized as effective materials with excellent underwater oleophobicity to resist oil fouling. ${ }^{35,50}$ The self-cleaning properties of the PSBMAmodified $\mathrm{Ca}^{2+}$-ISE were characterized by investigating the underwater-oil adhesion on the membrane surface. As illustrated in Figure 2A, the diesel oil droplet can quickly adhere to the surface of the pristine $\mathrm{Ca}^{2+}$-ISM once it contacts the membrane surface, indicating that the pristine $\mathrm{Ca}^{2+}$-ISE has a high oil-fouling tendency. In contrast, no obvious oil adhesion is observed for the PSBMA-modified $\mathrm{Ca}^{2+}$-ISM (Figure 2B). In addition, the free oil droplet can quickly slide away from the surface of PSBMA-modified $\mathrm{Ca}^{2+}$-ISM (Figure $2 \mathrm{C})$. Indeed, these data prove that the PSBMA-modified membrane has an excellent self-cleaning ability. After PSBMA modification, a zwitterionic membrane surface can be obtained which has excellent fouling resistance due to the formation of a compact and continuous hydration layer on the membrane surface via electrostatically induced hydration. ${ }^{13,39}$ The hydration layer could strongly weaken the hydrophobic interactions between the oil droplets and membrane surface. Under the conditions of slight hydraulic stirring, the oil droplets can be easily expelled from the membrane surface without any adhesion. In this case, the coalescence of oil droplets and formation of an oil layer on the membrane surface 
A
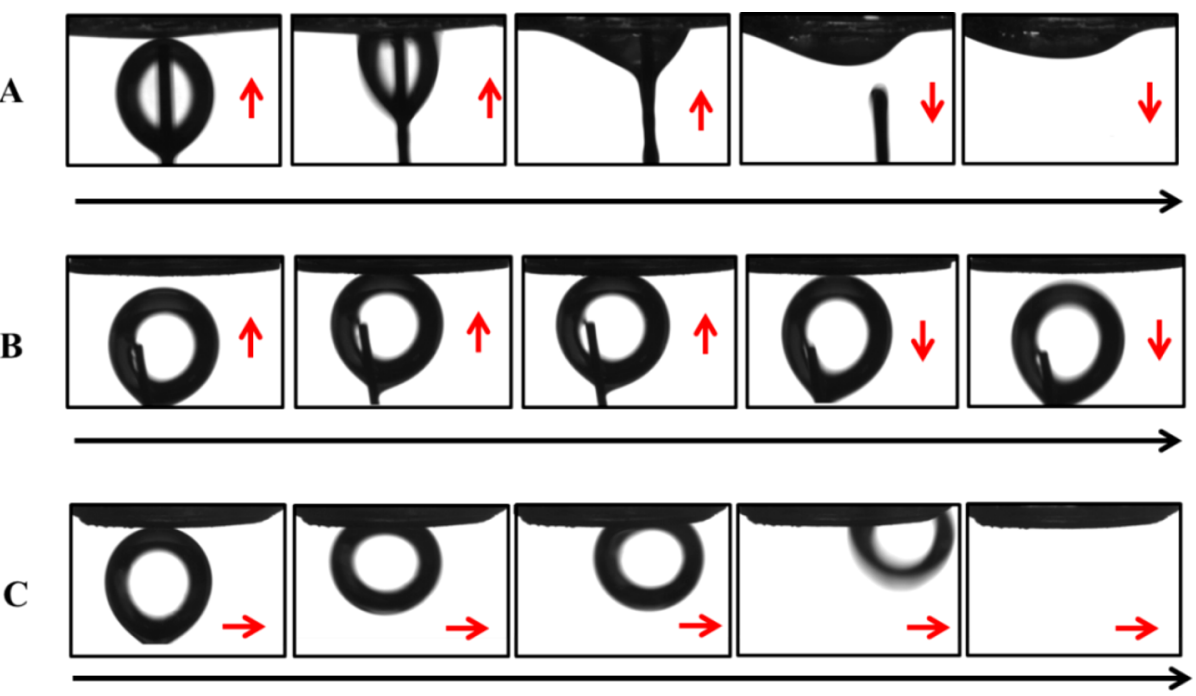

Figure 2. Self-cleaning properties of the PSBMA-modified $\mathrm{Ca}^{2+}$-selective membranes: (A) dynamic images of a diesel oil droplet approaching and adhering to the pristine $\mathrm{Ca}^{2+}$-selective membrane, (B) dynamic images of a diesel oil droplet approaching and leaving the PSBMA-modified Ca ${ }^{2+}$ selective membrane, and $(\mathrm{C})$ dynamic images of a free diesel oil droplet sliding away from the PSBMA-modified $\mathrm{Ca}^{2+}$-selective membrane. The red arrows represent the moving directions of the diesel oil droplets.

can be effectively inhibited. Therefore, the oil-fouling resistance of the PSBMA-modified membrane electrode can be significantly enhanced.

It should be noted that the improvement in sensor's foulingresistance properties should not sacrifice the analytical performance. $^{30}$ Therefore, the potentiometric responses of the pristine and PSBMA-modified $\mathrm{Ca}^{2+}$-ISEs were tested in the range from $10^{-8}$ to $10^{-2} \mathrm{M}$. As shown in Figure $3 \mathrm{~A}$, the
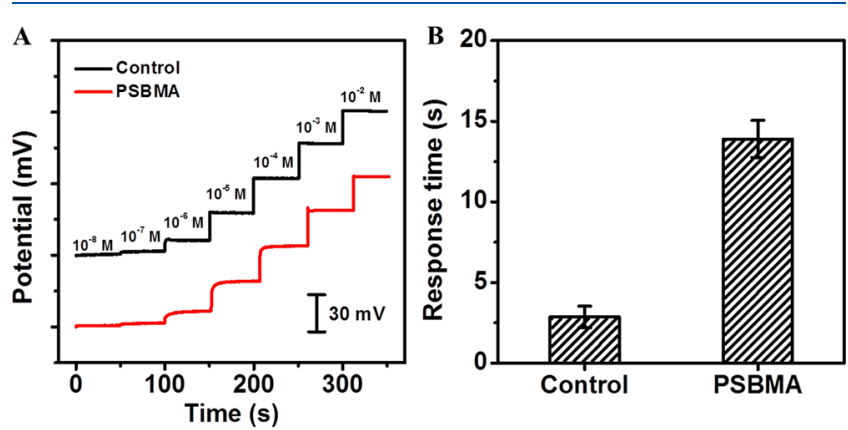

Figure 3. Influence of the surface modification on the sensor's analytical performance: (A) time-dependent potentiometric response traces of the pristine and PSBMA-modified $\mathrm{Ca}^{2+}$-ISEs and (B) response times of the pristine and PSBMA-modified $\mathrm{Ca}^{2+}$-ISEs. The response times were calculated from the potentiometric response traces when the concentration of $\mathrm{Ca}^{2+}$ was changed from $1 \times 10^{-6}$ to $1 \times 10^{-5} \mathrm{M}$. Error bars represent one standard deviation for three measurements.

PSBMA modification has little influence on the sensor's performance in terms of linear range, response slope, and detection limit. The pristine $\mathrm{Ca}^{2+}$-ISE exhibits a Nernstian response slope of $29.2 \pm 0.2 \mathrm{mV}$ decade $^{-1}$ over the range from $10^{-6}$ to $10^{-2} \mathrm{M}$ with a detection limit of $5.3 \times 10^{-7} \mathrm{M}$. Similar to the pristine $\mathrm{Ca}^{2+}$-ISE, the PSBMA-modified $\mathrm{Ca}^{2+}$-ISE shows a response slope of $28.7 \pm 0.9 \mathrm{mV}$ decade $^{-1}$ with a detection limit of $5.5 \times 10^{-7} \mathrm{M}$. These results indicate that the PSBMA coating cannot impair the thermodynamic extraction equilibrium of $\mathrm{Ca}^{2+}{ }^{21}$ As shown in Figure 3B, the response time of the PSBMA-modified $\mathrm{Ca}^{2+}$-ISE is longer than that of the pristine $\mathrm{Ca}^{2+}$-ISE. After modification, the response time of the $\mathrm{Ca}^{2+}$-ISE increases from 2.9 to $13.9 \mathrm{~s}$ when the concentration of $\mathrm{Ca}^{2+}$ is changed from $10^{-6}$ to $10^{-5} \mathrm{M}$. Such a change in response time is more pronounced for lower concentrations of $\mathrm{Ca}^{2+}$ but negligible for concentrations higher than $10^{-3} \mathrm{M}$. This may be attributed to the fact that the PSBMA layer could prevent the diffusion of $\mathrm{Ca}^{2+}$ between the sensing membrane and the aqueous layer. ${ }^{20,52}$ The selectivity coefficients of the PSBMA-modified $\mathrm{Ca}^{2+}$-ISE were measured by using the separate solution method, ${ }^{46}$ and $\mathrm{Na}^{+}, \mathrm{K}^{+}$, and $\mathrm{Mg}^{2+}$ were selected as the interfering ions. The results shown in Table S1 demonstrate that the selectivity coefficients of the PSBMAmodified $\mathrm{Ca}^{2+}$-ISE are comparable to those of the pristine $\mathrm{Ca}^{2+}$-ISE.

In order to evaluate the feasibility of the proposed selfcleaning $\mathrm{Ca}^{2+}$-ISE for practical applications, the potentiometric responses of the pristine and PSBMA-modified $\mathrm{Ca}^{2+}$-ISEs before and after immersion of the electrodes in the oilcontaining wastewaters were measured. As illustrated in Figure $4 \mathrm{~A}$, after immersion in the wastewater containing $5 \%(\mathrm{v} / \mathrm{v})$ diesel oil for $1 \mathrm{~h}$ under stirring, the response slope of the pristine $\mathrm{Ca}^{2+}$-ISE decreases by $56.2 \%$ (from 29.2 to $12.8 \mathrm{mV}$ decade $\left.^{-1}\right)$. In contrast, no obvious change is observed for the PSBMA-modified $\mathrm{Ca}^{2+}$-ISE (from 28.7 to $28.4 \mathrm{mV} \mathrm{decade}^{-1}$ ).
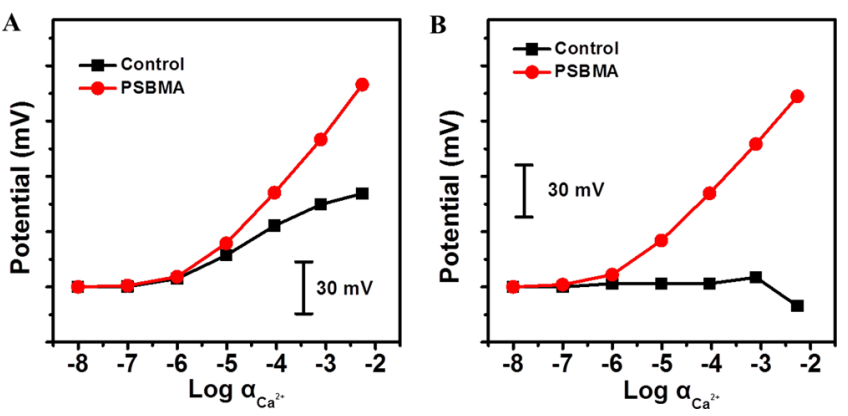

Figure 4. Typical calibration curves of the pristine and PSBMAmodified $\mathrm{Ca}^{2+}$-ISEs after underwater contacts with $5 \%(\mathrm{v} / \mathrm{v})$ diesel oil (A) and crude oil (B), respectively, for $1 \mathrm{~h}$ under stirring. 
The similar results are also observed for the crude oil fouling. As shown in Figure 4B, the pristine $\mathrm{Ca}^{2+}$-ISE even loses its original potential response, while the PSBMA-modified $\mathrm{Ca}^{2+}$ ISE still maintains an acceptable response slope of $26.0 \mathrm{mV}$ decade $^{-1}$. These results suggest that the proposed self-cleaning $\mathrm{Ca}^{2+}$-ISE exhibits a significantly improved stability when exposed to oil foulants. Such remarkable difference in sensor's potentiometric response is probably due to the fact that the pristine PVC-based oleophilic membrane has a tendency to adsorb oil droplets resulting in the accumulation of oils on the membrane surface. ${ }^{13}$ The adsorbed oil foulants could be dissolved in the membrane phase to change the membrane components and/or may form an oil layer to insulate the ISE membrane from the sample solution. For the PSBMA-modified $\mathrm{Ca}^{2+}$-ISE, the self-cleaning oleophobic coating can effectively prevent the adhesion of oil foulants on the electrode surface and thus improve the sensor's analytical stability when exposed to oil foulants.

Furthermore, the $\mathrm{Ca}^{2+}$-ISMs were examined visually after contact with the oil-containing wastewaters. The diesel oil sample was stained using Sudan III to improve visualization. Indeed, as shown in Figure S6, after contacts with the oilcontaining wastewaters, a large amount of the tested oils with the dense and uniform distributions can be observed on the unmodified $\mathrm{Ca}^{2+}$-ISMs, indicating the high fouling tendency of the unmodified membrane. Figure S6 also indicates that, for the PSBMA-modified $\mathrm{Ca}^{2+}$-ISMs, negligible oil adhesion is observed after contacts with the oil-containing wastewaters, thus further confirming the enhanced fouling-resistance of the proposed sensor.

\section{CONCLUSIONS}

We demonstrate here for the first time that the oil-fouling resistance of polymeric membrane $\mathrm{Ca}^{2+}$-ISEs can be enhanced by surface modification of a zwitterionic PSBMA coating. Experiments show that the analytical performance of the $\mathrm{Ca}^{2+}$ ISE in terms of response slope, detection limit, and selectivity is not influenced by the PSBMA coating. The PSBMA coating increases the UWOCA of the sensor's surface from $25.4 \pm 2.1$ to $141.5 \pm 3.4^{\circ}$ and endows the sensor with excellent selfcleaning properties against oil foulants. After fouling with $5 \%$ $(\mathrm{v} / \mathrm{v})$ diesel oil or crude oil, the response of the pristine $\mathrm{Ca}^{2+}$ ISE decreases by $56.2 \%$ or even completely lost in terms of the response slope. In contrast, the PSBMA-modified $\mathrm{Ca}^{2+}$-ISEs can still maintain acceptable response slopes after being fouled by oils. The proposed strategy has a promising potential to develop polymeric membrane ISEs with excellent oil-fouling resistance for reliable potentiometric detection in the oilpolluted environment.

\section{ASSOCIATED CONTENT}

\section{SI Supporting Information}

The Supporting Information is available free of charge at https://pubs.acs.org/doi/10.1021/acs.analchem.1c01116.

Experimental details including reagents and characterization; SEM images (Figure S1) for the PSBMAmodified membrane; water contact angles (Figure S2) for the PSBMA-modified membrane; ATR-FTIR spectra for the polydopamine and the product from polymerization of dopamine and SBMA (Figure S3); influences of the modification time on the UWOCA for PSBMAmodified $\mathrm{Ca}^{2+}$-selective membrane (Figure $\mathrm{S} 4$ ); UWO-
CAs for the polydopamine-modified $\mathrm{Ca}^{2+}$-selective membrane (Figure S5); photographs of the pristine and PSBMA-modified $\mathrm{Ca}^{2+}$-selective membranes before and after contact with oils (Figure S6); and potentiometric selectivity coefficients of $\mathrm{Ca}^{2+}$-ISEs before and after modification (Table S1) (PDF)

\section{AUTHOR INFORMATION}

\section{Corresponding Author}

Wei Qin - CAS Key Laboratory of Coastal Environmental Processes and Ecological Remediation, Yantai Institute of Coastal Zone Research (YIC), Chinese Academy of Sciences (CAS), Shandong Key Laboratory of Coastal Environmental Processes YICCAS, Yantai, Shandong 264003, P. R. China; Laboratory for Marine Biology and Biotechnology, Pilot National Laboratory for Marine Science and Technology (Qingdao), Qingdao, Shandong 266237, P. R. China; Center for Ocean Mega-Science, Chinese Academy of Sciences, Qingdao, Shandong 266071, P. R. China; 1 orcid.org/ 0000-0002-9606-7730; Email: wqin@yic.ac.cn; Fax: +86$535-2109000$

\section{Authors}

Longbin Qi - CAS Key Laboratory of Coastal Environmental Processes and Ecological Remediation, Yantai Institute of Coastal Zone Research (YIC), Chinese Academy of Sciences (CAS), Shandong Key Laboratory of Coastal Environmental Processes YICCAS, Yantai, Shandong 264003, P. R. China; University of Chinese Academy of Sciences, Beijing 100049, P. R. China

Tianjia Jiang - CAS Key Laboratory of Coastal Environmental Processes and Ecological Remediation, Yantai Institute of Coastal Zone Research (YIC), Chinese Academy of Sciences (CAS), Shandong Key Laboratory of Coastal Environmental Processes YICCAS, Yantai, Shandong 264003, P. R. China; Laboratory for Marine Biology and Biotechnology, Pilot National Laboratory for Marine Science and Technology (Qingdao), Qingdao, Shandong 266237, P. R. China

Rongning Liang - CAS Key Laboratory of Coastal Environmental Processes and Ecological Remediation, Yantai Institute of Coastal Zone Research (YIC), Chinese Academy of Sciences (CAS), Shandong Key Laboratory of Coastal Environmental Processes YICCAS, Yantai, Shandong 264003, P. R. China; Laboratory for Marine Biology and Biotechnology, Pilot National Laboratory for Marine Science and Technology (Qingdao), Qingdao, Shandong 266237, P.

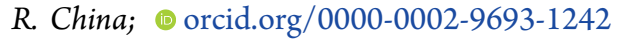

Complete contact information is available at: https://pubs.acs.org/10.1021/acs.analchem.1c01116

\section{Author Contributions}

\#Longbin Qi and Tianjia Jiang contributed equally to this work.

Notes

The authors declare no competing financial interest.

\section{ACKNOWLEDGMENTS}

This work was financially supported by the National Natural Science Foundation of China (Grants U2006208, 41806119, and 21874151), the Key Deployment Project of Centre for Ocean Mega-Research of Science, Chinese Academy of 
Sciences (Grant COMS2020J06), the National Key Research and Development Program of China (Grant 2016YFC1400700), the Youth Innovation Promotion Association of CAS (Grant 2014190), the Taishan Scholar Program of Shandong Province (Grants tspd20181215 and tsqn202103132), and the Science and Technology Project of Yantai City (Grant 2020MSGY051).

\section{REFERENCES}

(1) Zdrachek, E.; Bakker, E. Anal. Chem. 2019, 91, 2-26.

(2) Liang, R. N.; Wang, Q. W.; Qin, W. Sens. Actuators, B 2015, 208, 267-272.

(3) Buhlmann, P.; Pretsch, E.; Bakker, E. Chem. Rev. 1998, 98, $1593-1687$.

(4) Crespo, G. A. Electrochim. Acta 2017, 245, 1023-1034.

(5) Zuliani, C.; Diamond, D. Electrochim. Acta 2012, 84, 29-34.

(6) Peterson, C. H.; Rice, S. D.; Short, J. W.; Esler, D.; Bodkin, J. L.; Ballachey, B. E.; Irons, D. B. Science 2003, 302, 2082-2086.

(7) Blumer, M.; Sass, J. Science 1972, 176, 1120-1122.

(8) Martinez-Gomez, C.; Vethaak, A. D.; Hylland, K.; Burgeot, T.; Koehler, A.; Lyons, B. P.; Thain, J.; Gubbins, M. J.; Davies, I. M. ICES J. Mar. Sci. 2010, 67, 1105-1118.

(9) Magalhaes, K. M.; Barros, K. V. d. S.; Lima, M. C. S. d.; RochaBarreira, C. d. A.; Rosa Filho, J. S.; Soares, M. d. O. Sci. Total Environ. 2021, 764, 142872.

(10) Yu, J.; Zhou, D.; Yu, M.; Yang, J.; Li, Y.; Guan, B.; Wang, X.; Zhan, C.; Wang, Z.; Qu, F. Sci. Total Environ. 2021, 765, 142694142694.

(11) Al Shami, A.; Harik, G.; Alameddine, I.; Bruschi, D.; Garcia, D. A.; El-Fadel, M. Sci. Total Environ. 2017, 574, 234-245.

(12) Lorant, S.; Bohnke, O.; Breviere, J.; Bohnke, C. Electroanalysis 2013, 25, 223-229.

(13) Zhang, R. N.; Liu, Y. N.; He, M. R.; Su, Y. L.; Zhao, X. T.; Elimelech, M.; Jiang, Z. Y. Chem. Soc. Rev. 2016, 45, 5888-5924.

(14) Asatekin, A.; Mayes, A. M. Environ. Sci. Technol. 2009, 43, 4487-4492.

(15) Zhao, Y.; Yang, X.; Yan, L.; Bai, Y.; Li, S.; Sorokin, P.; Shao, L. J. Membr. Sci. 2021, 618, 118525.

(16) Mubita, T.; Porada, S.; Aerts, P.; van der Wal, A. J. Membr. Sci. 2020, 607, 118000.

(17) Buhlmann, P.; Hayakawa, M.; Ohshiro, T.; Amemiya, S.; Umezawa, Y. Anal. Chem. 2001, 73, 3199-3205.

(18) Espadas Torre, C.; Meyerhoff, M. E. Anal. Chem. 1995, 67, 3108-3114.

(19) Soto, R. J.; Hall, J. R.; Brown, M. D.; Taylor, J. B.; Schoenfisch, M. H. Anal. Chem. 2017, 89, 276-299.

(20) Jiang, X. J.; Wang, P.; Liang, R. N.; Qin, W. Anal. Chem. 2019, 91, 6424-6429.

(21) Espadas-Torre, C.; Oklejas, V.; Mowery, K.; Meyerhoff, M. E. J. Am. Chem. Soc. 1997, 119, 2321-2322.

(22) Frost, M. C.; Rudich, S. M.; Zhang, H. P.; Maraschio, M. A.; Meyerhoff, M. E. Anal. Chem. 2002, 74, 5942-5947.

(23) Zhang, Q.; Murray, G. P.; Hill, J. E.; Harvey, S. L.; Rojas-Pena, A.; Choi, J.; Zhou, Y.; Bartlett, R. H.; Meyerhoff, M. E. Anal. Chem. 2020, 92, 13641-13646.

(24) Jiang, T. J.; Qi, L. B.; Qin, W. Anal. Chem. 2019, 91, 1326813274.

(25) Jiang, T. J.; Qi, L. B.; Hou, C.; Fang, S. T.; Qin, W. Anal. Chem. 2020, 92, 12132-12136.

(26) Qi, L. B.; Jiang, T. J.; Liang, R. N.; Qin, W. Sens. Actuators, B 2021, 328, 129014.

(27) Phillips, F.; Kaczor, K.; Gandhi, N.; Pendley, B. D.; Danish, R. K.; Neuman, M. R.; Toth, B.; Horvath, V.; Lindner, E. Talanta 2007, $74,255-264$.

(28) Lisak, G.; Cui, J.; Bobacka, J. Sens. Actuators, B 2015, 207, $933-$ 939.

(29) Ding, J.; He, N.; Lisak, G.; Qin, W.; Bobacka, J. Sens. Actuators, B 2017, 243, 346-352.
(30) Pawlak, M.; Bakker, E. Electroanalysis 2014, 26, 1121-1131.

(31) Pawlak, M.; Grygolowicz-Pawlak, E.; Crespo, G. A.; Mistlberger, G.; Bakker, E. Electroanalysis 2013, 25, 1840-1846.

(32) Lakshmipriya, T.; Horiguchi, Y.; Nagasaki, Y. Analyst 2014, 139, 3977-3985

(33) Berrocal, M. J.; Johnson, R. D.; Badr, I. H. A.; Liu, M.; Gao, D.; Bachas, L. G. Anal. Chem. 2002, 74, 3644-3648.

(34) Xu, Z. Y.; Han, R.; Liu, N. Z.; Gao, F. X.; Luo, X. L. Sens. Actuators, B 2020, 319, 128253.

(35) Chen, S. Q.; Xie, Y.; Chinnappan, A.; Wei, Z. W.; Gu, Q. L.; He, H. Y.; Fang, Y. L.; Zhang, X.; Lakshminarayanan, R.; Zhao, W. F.; Zhao, C. S.; Ramakrishna, S. Sci. Total Environ. 2020, 729, 138876.

(36) Zhang, S.; Ly, Q. V.; Nghiem, L. D.; Wang, J.; Li, J.; Hu, Y. J. Membr. Sci. 2020, 596, 117748.

(37) Jiang, S. Y.; Cao, Z. Q. Adv. Mater. 2010, 22, 920-932.

(38) Shao, Q.; He, Y.; Jiang, S. Y. J. Phys. Chem. B 2011, 115, 83588363.

(39) Wu, J.; Lin, W. F.; Wang, Z.; Chen, S. F.; Chang, Y. Langmuir 2012, 28, 7436-7441.

(40) Feng, T. T.; Ji, W. L.; Zhang, Y.; Wu, F.; Tang, Q.; Wei, H.; Mao, L. Q.; Zhang, M. N. Angew. Chem., Int. Ed. 2020, 59, 2344523449.

(41) Bobacka, J. Anal. Chem. 1999, 71, 4932-4937.

(42) Mousavi, Z.; Bobacka, J.; Lewenstam, A.; Ivaska, A. J. Electroanal. Chem. 2009, 633, 246-252.

(43) Zhang, C.; Ma, M. Q.; Chen, T. T.; Zhang, H.; Hu, D. F.; Wu, B. H.; Ji, J.; Xu, Z. K. ACS Appl. Mater. Interfaces 2017, 9, 3435634366.

(44) Lee, H.; Dellatore, S. M.; Miller, W. M.; Messersmith, P. B. Science 2007, 318, 426-430.

(45) Ryu, J. H.; Messersmith, P. B.; Lee, H. ACS Appl. Mater. Interfaces 2018, 10, 7523-7540.

(46) Bakker, E. J. Electrochem. Soc. 1996, 143, L83-L85.

(47) Liu, Y. L.; Ai, K. L.; Lu, L. H. Chem. Rev. 2014, 114, 50575115.

(48) Mrowczynski, R.; Markiewicz, R.; Liebscher, J. Polym. Int. 2016, $65,1288-1299$.

(49) Zhang, Y. Q.; Cheng, X. Q.; Jiang, X.; Urban, J. J.; Lau, C. H.; Liu, S. Q.; Shao, L. Mater. Today 2020, 36, 40-47.

(50) He, K.; Duan, H. R.; Chen, G. Y.; Liu, X. K.; Yang, W. S.; Wang, D. Y. ACS Nano 2015, 9, 9188-9198.

(51) Xu, Y.; Xu, C.; Shvarev, A.; Becker, T.; De Marco, R.; Bakker, E. Anal. Chem. 2007, 79, 7154-7160.

(52) Muslinkina, L.; Pretsch, E. Chem. Commun. 2004, No. 10, $1218-1219$. 